\title{
Experimental Study of the Separation of Oil in Water Emulsions by Tangential Flow Microfiltration Process. Part 1: Analysis of Oil Rejection Efficiency and Flux Decline
}

Wai Lam Loh ${ }^{1,2}$, Thiam Teik Wan ${ }^{1,2 *}$, Vivek Kolladikkal Premanadhan ${ }^{1}$, Ko Ko Naing ${ }^{1,2}$, Nguyen Dinh Tam ${ }^{1,2}$, Valente Hernandez Perez ${ }^{1,2}$ and Yu Qiao Zhao'

${ }^{1}$ Department of Mechanical Engineering, Faculty of Engineering, National University of Singapore, Singapore

${ }^{2}$ Centre for Offshore Research and Engineering (CORE), National University of Singapore, Singapore

\begin{abstract}
Management of produced water is a major issue offshore. Microfiltration has emerged as a useful alternative for treating the oil-water emulsions to meet the regulatory limit for disposal. In this work, tangential flow (cross flow) microfiltration of oil-water mixture was studied. The tangential flow microfiltration process was investigated using a ceramic membrane of $0.5 \mu \mathrm{m}$ pore size. For this phase of work, medium viscosity paraffin oil was used as substitute to crude oil. Using oily water feed of $500-1000$ ppm oil concentration, a microfiltration ceramic membrane of 0.5 $\mu \mathrm{m}$ pore size was proven capable of producing a high purity filtrate lower than the threshold required for offshore produced water effluent, typically $29 \mathrm{mg} / \mathrm{l}$ residual oil, in the Gulf of Mexico. However, membrane has a major drawback in the form of fouling. Decline in permeation flux should be expected over time of operation. This limitation has certainly impeded the large scale applications of microfiltration process in the field of oil and gas processing industry. An optimized cleaning process is needed to restore membrane performance.
\end{abstract}

Keywords: Microfiltration; Oil in water emulsions; Residual oil; Permeability; Oil rejection efficiency

\section{Introduction}

Oilfield produced water treating equipment in use today is designed to remove discrete droplets of oil from the water phase. Conventional equipment such as gravity separator and hydro-cyclone separates the dispersed phases from continuous phases according to their density difference under the action of gravity force or centrifugal force induced by swirling flow. As flows move through chokes, valves, pumps, or other constrictions during crude oil production, the droplets can be torn into smaller droplets by the pressure differential across the devices. These small droplets can be further stabilized in the water by surfactants. The addition of excess production chemicals (such as surfactants) forms an encapsulation of hydrophilic and/or hydrophobic particles on the oil droplets, as shown in Figure 1.

It reduces the interfacial tension so that the coalescence and separation of small droplets become extremely difficult. The inherent difficulty of coalescing emulsified oils in the conventional separating equipment, and ineffective separation of finer emulsion droplets slightly

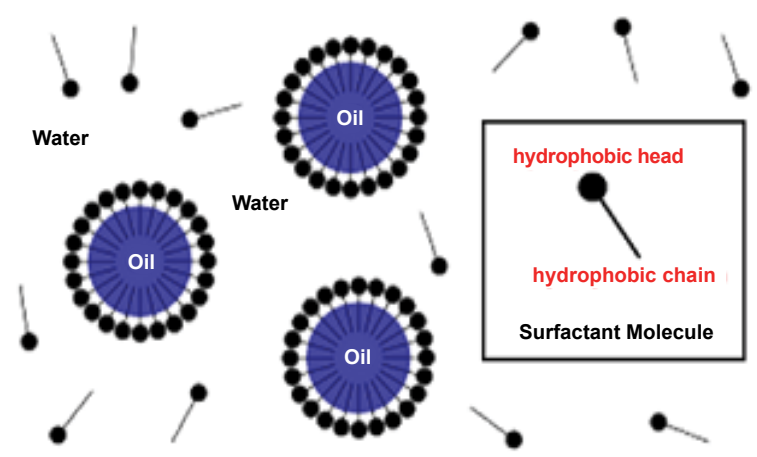

Figure 1: Oil emulsion droplets are being stabilized with surfactant molecules. lighter than the continuous phase, makes the conventional separation techniques difficult to treat oily effluent which contains fine emulsions. The use of microfiltration technology is becoming an increasingly popular alternation for the separation of emulsified oil in lieu of the conventional separation approaches.

Membrane separation such as microfiltration (MF), ultra-filtration (UF), nano-filtration (NF), and reverse osmosis (RO) can be used to separate different sized materials [1]. A considerable amount of experimental works and theoretical modelling studies [1-7] in the past two decades that have made possible for the use of low pressure driven membranes for MF of membrane pore size between 0.1 to $5 \mu \mathrm{m}$. UF with membrane pore size less than $0.1 \mu \mathrm{m}$ or a combination of $\mathrm{MF} / \mathrm{UF}$ polymeric or ceramic membranes are suitable for removing oil content of oilfield produced water. This method involves using low pressure to force the continuous phase to permeate through a membrane into the discharge.

The rejection efficiencies are controlled primarily by the choice of the membrane pore size and not by the difference of density in between the dispersed phase and continuous phase. Because of the many unique properties of the membrane technologies such as no phase change, no chemical addition and simple operation, membrane processes usually provide a better option over traditional separation method in oil and gas processing industries.

*Corresponding author: Wan Thiam Teik, Research Associate, Faculty of Engineering, National University of Singapore, Singapore, Tel: 65-6516-8076; Fax: 65-6779-1936; E-mail: mpewtt@nus.edu.sg

Received May 21, 2014; Accepted August 21, 2014; Published August 26, 2014

Citation: Loh WL, Wan TT, Premanadhan VK, Naing KK, Tam ND, et al. (2014) Experimental Study of the Separation of Oil in Water Emulsions by Tangential Flow Microfiltration Process. Part 1: Analysis of Oil Rejection Efficiency and Flux Decline. J Membra Sci Technol 5: 130. doi:10.4172/2155-9589.1000130

Copyright: ( 2014 Loh WL, et al. This is an open-access article distributed under the terms of the Creative Commons Attribution License, which permits unrestricted use, distribution, and reproduction in any medium, provided the original author and source are credited. 
Citation: Loh WL, Wan TT, Premanadhan VK, Naing KK, Tam ND, et al. (2014) Experimental Study of the Separation of Oil in Water Emulsions by Tangential Flow Microfiltration Process. Part 1: Analysis of Oil Rejection Efficiency and Flux Decline. J Membra Sci Technol 5: 130. doi:10.4172/2155-9589.1000130

Page 2 of 6

Koltuniewicz et al. recommended separating oil emulsion from water using ultra/microfiltration performed with ceramic or certain hydrophilic polymeric membranes [5]. Ceramic membranes are often preferred over delicate polymeric membrane because the former have better tolerance to high temperature, high oil contents, foulants, and strong cleaning agents [1]. Mueller et al. proved that microfiltration ceramic membranes at 0.2 and $0.8 \mu \mathrm{m}$ pore sizes are capable of producing a very high quality permeate from a feed concentrations around 250-1000 ppm of crude oil with droplets size range of 1-10 $\mu \mathrm{m}$. It was reported to produce a very high quality permeate, containing lower than $6 \mathrm{ppm}$ of total hydrocarbons in the permeate sample [2]. Chen et al. also tested the performance of ceramic tangential flow (cross-flow) microfiltration to separate oil, grease, and suspended solids from produced water. Permeate quality of dispersed oil and grease was $5 \mathrm{mg} / \mathrm{L}$ and of suspended solids was less than $1 \mathrm{mg} / \mathrm{L}$ [6]. Despite these advances, very few applications of this technology in the separation of oil water mixture have been implemented especially for offshore processing. Although many pilot tests have been conducted using membranes for filtering produced water but with limited success due to their propensity to foul irreversibly with oil and dirt [8].

This research sought to conduct a series of experiments for investigating the characteristics of tangential flow microfiltration of oil-in-water emulsions, and factors determining the membrane performance, as well as the study of fouling control measures. The primary objective of this work is to answer a research question whether tangential flow microfiltration is able to reduce the residual content of the produced water effluent to a level lower than maximum threshold as required for a discharge in sea as claimed in several published works [1-7].

\section{Theory}

The permeation flux across the membrane is calculated from $\mathrm{J}=\mathrm{V} /$ $\left(\mathrm{A}^{*} \mathrm{t}\right)$ where $\mathrm{J}$ is the liquid flux across the membrane, A the membrane surface area in contact with the liquid, $t$ the run time of the experiment, and $\mathrm{V}$ the volume of permeate collected during time $\mathrm{t}$. The rate of permeate flux through membrane filter is dictated by the resistance of the filter to the flow of fluid and any resistance associated with the trapped particulate. The basic Darcy's Law is defined as:

$$
\frac{d p}{d x}=\frac{\mu u}{K}
$$

where $\mathrm{dp}=\Delta \mathrm{P}$ as the pressure drop across the filter, $\mu$ as the viscosity of the permeate fluid, $d x$ is the thickness of the filter, $K$ as the permeability, and $\mathrm{u}=\mathrm{J}$ as permeation flux through the filter. Equation $(2-1)$ is rearranged to give a general equation as:

$$
J=\frac{K \Delta P}{\mu \Delta x}
$$

Permeability K refers to the ease with which water can flow through a filter. The resistance to permeation of a membrane is a function of the membrane pore size, feed stream components, and the degree to which gel layer formation and fouling layer formation occur. Increasing the feed stream circulation rate will, as a general rule, reduce gel layer thickness and increase flux.

Darcy's Law states that the flux is directly proportional to the potential pressure drop and inversely proportional to the resistance, R:

$$
R=\frac{\Delta P}{J}=\mu R_{t}
$$

At a constant applied pressure for a given feed with constant composition and flow viscosity, the filtration flux is inversely proportional to the total resistance Rt. The total resistance can be expressed as follows [9]:

$$
R_{t}=R_{m}+R_{c}+R_{c p}+R_{g}
$$

The total resistance consists of resistance by the filter media $\left(\mathrm{R}_{\mathrm{m}}\right)$ which can include the pore blocking resistance $\left(R_{p}\right)$ and resistance by adsorption $\left(\mathrm{R}_{\mathrm{a}}\right)$; resistance due to internal colloidal fouling $\left(\mathrm{R}_{\mathrm{c}}\right)$; resistance due to formation of a highly concentrated layer adjacent to the membrane, concentration polarization $\left(\mathrm{R}_{\mathrm{cp}}\right)$; and resistance caused by the formation of the gel layer $\left(\mathrm{R}_{\mathrm{g}}\right)$, due to the increasing concentration of particles near the surface of the membrane.

The oil rejection efficiency $\left(\mathrm{R}_{\mathrm{O}}\right)$ of the filter is defined as the ability to retain dispersed oil phase from flowing across the membrane:

$$
R_{o}=1-\frac{C_{P}}{C_{F}}
$$

where $C_{P}$ and $C_{F}$ are the measured oil concentration of permeate and feed, respectively. For instances, oil rejection efficiency of at least $97 \%$ is anticipated for any filter in order to meet a permeation quality of not exceeding $30 \mathrm{ppm}$ of residual oil, assuming a feed stream of 1000 ppm oil concentration.

\section{Methodology}

A two phase test rig was built to conduct experiments on the separation of oil-in-water emulsions using tangential flow microfiltration. The experimental set up is shown schematically in Figure 2.

The experimental setup consists of an emulsion feed tank equipped with mixer, clean water tank, centrifugal feed pump, tangential flow microfiltration module, vortex flow meters, pressure gauges, thermo sensors, valves and piping. A ceramic membrane of $0.5 \mu \mathrm{m}$ pore size was initially back-flushed with clean water. The contaminated oily water was flushed to the emulsion tank to avoid contamination of the clean water tank. After the cleaning, the oil in water feed was pumped into the microfiltration module for the experiments.

The separation of the oil from water is achieved by applying atmospheric pressure to the permeate side of the membrane whilst the other side is exposed to the pressurized liquid to be separated. When the feed stream flows through the membrane bounded channel, it splits into two streams, namely, retentate and permeate (or filtrate). The retentate was returned to the feed tank whilst the filtrate was collected for measurement of residual oil content. The feed flow rate to membrane module was regulated by varying the rate of flows returning to the feed tank, meanwhile, the desirable trans-membrane pressure was obtained by varying both the feed and choke valve on the retentate side.

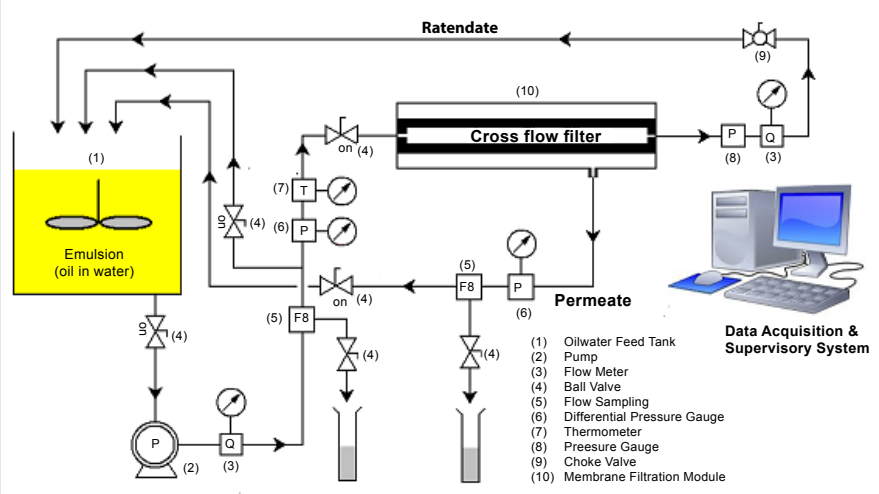

Figure 2: Schematic sketch of the experimental set-up. 
Citation: Loh WL, Wan TT, Premanadhan VK, Naing KK, Tam ND, et al. (2014) Experimental Study of the Separation of Oil in Water Emulsions by Tangential Flow Microfiltration Process. Part 1: Analysis of Oil Rejection Efficiency and Flux Decline. J Membra Sci Technol 5: 130. doi:10.4172/2155-9589.1000130

Page 3 of 6

\begin{tabular}{|c|c|c|c|}
\hline Liquid & Density $\left(\mathrm{kg} / \mathrm{m}^{3}\right)$ at $20^{\circ} \mathrm{C}$ & Viscosity (cP) at $20^{\circ} \mathrm{C}$ & API \\
\hline Clean water & 998 & 1.02 & 10 \\
\hline Paraffin oil & 905 & 87.12 & 25.54 \\
\hline
\end{tabular}

Table 1: Physical properties of liquids used in the experiments.

When the feed flows through the filter tube, oil droplets tend to deposits on the membrane, but they were continuously removed by the feed flowing tangentially across the surface of the membrane. Constant concentration of the feed is virtually maintained by returning permeate and retentate to the emulsion feed tank. Only a small amount of permeate samples were withdrawn for the measurement of residual content. As the permeate volume compared with the feed volume are small, the feed concentration was considered to be unchanged.

In the experiments, paraffin oil which represents moderate viscosity oil was used as substitute to crude oil. Colored liquid paraffin was used to enable visual inspection during the preparation of emulsion. The dynamic viscosity of paraffin oil used in the experiments was determined using the HAAKE MARS rotational rheometer and the physical properties of the liquids used in the experiments are shown in Table 1.

The oily water mixture was prepared by emulsifying the desirable percentage of paraffin oil in clean water (500 and $1000 \mathrm{ppm}$ ). The oil-water mixture was agitated using a mixer for at least five minutes, further re-circulated to the emulsion feed tank by a centrifugal feed pump until a milky white mixture was formed. The result was stable oil in water emulsions which doesn't separate by gravity for days or weeks. However, emulsions is shear sensitive, therefore the retentate was recirculated to the feed tank, where they were continuously stirred with an emulsifier mixer to prevent oil droplets from coalescing to ensure homogeneity throughout the experiments.

All the microfiltration experiments were conducted in a series of batch runs with trans-membrane pressure of 1.0, 1.5, 2.0, 2.5, 3.0 and $3.5 \mathrm{bar}(\mathrm{g})$, respectively. The starting trans-membrane pressure was initiated from 1 bar(g), and gradually increased by a step of 0.5 bar(g) at each run, until a final pressure of $3 \mathrm{bar}(\mathrm{g})$ was reached during the fifth runs. To this end, the entire cycle was repeated thereafter. Each batch (run) of experiments took a ten minutes interval, sum up to an approximate total time of hundred fifty minutes per fifteen runs of experiments, afterward, then clean water feed was replaced by oilwater feed of $1000 \mathrm{ppm}$ concentration for the study on oil rejection performance. Next, the experiments were repeated with oil-water feed of 500 ppm concentration.

The permeate quality was monitored by collecting samples at different time intervals for the measurement of oil concentration in unit of ppm (part per million) by volume. The sampling approach here involved the collection of representative samples of the emulsion from the pipelines and subsequent analysis of the samples using solvent extraction method. Solvent extraction method is often used to determine the oil content of dilute oil in water emulsion with concentrations as much as $1000 \mathrm{ppm}$ or so [10]. In this work, a TD-500D fluoro-meter was used to measure oil content in water samples by the detection of the fluorescent light emitted by oil that has been extracted into $\mathrm{n}$-hexane. The UV fluorescence value in TD-500D meter was then calibrated to known concentration of oil in water sample to give results equivalent to EPA method 1664 [11]. The fluorescence technique requires calibration by measuring the intensity of fluorescent light that is generated by a known concentration of hydrocarbon. Hence, the fluorescence values in the fluoro-meter were calibrated with respect to a known concentration of oil in water sample, as well as the distilled water sample (two points calibration, plus the mid-point calibration for a linearity check). The detection range is in between 5-1000 ppm of residual oil content.

During the experiments, all permeate samples collected were immediately photographed for visual comparison. All six samples showed no visible free phase of oil in permeate water. The crystal clear permeate quality gave good indication of effective rejection of oil emulsion after the filtration. However, it was difficult to justify the difference in oil concentration until they were later analyzed by fluorometer.

\section{Results and Discussions}

The aim of the experiment is to study the oil rejection efficiency under influence of pressure for initial evaluation of the potential application of ceramic microfiltration membrane in filtering oil phase from the oily water. The understanding of oil rejection efficiency under optimum operating conditions was important as a first step in the development of future processing equipment.

Two sets of experiment with a feed solution of different oil concentrations of 500 and $1000 \mathrm{ppm}$ were conducted. Both the experiments were run at approximate similar range of permeability values for fair comparison of results. For evaluation of the process and economic viability of membrane-based filtration applications, flux stability is a significant component which must be taken into consideration.

Fouling, not surprisingly, is frequently cited as the most important factor limiting the utilization of membranes in waste water treatment. It would affect the observation of filtration performance if was not addressed carefully. In the experiments, the first 15 runs uses only clean water as feed for monitoring the effects of trans-membrane pressure on permeability $(\mathrm{K}=\mathrm{J} \mu \Delta \mathrm{x} / \Delta \mathrm{P})$, while observing the fouling characteristics of membrane over a history of experiments order. In Figure 4, the ceramic membrane experienced a rapid decline in permeability in the beginning.

The rapid decline in permeability may be connected with the build-up of the concentration polarization layer and pore blocking mechanism. This phenomenon is inherent of all membrane filtration processes. A higher pressure causes the suspended impurities to rapidly deposited on the membrane surface, and the formation of cake increases the resistance to permeate flow and thus reduces the permeate flux and membrane performance, as illustrated in Figure 3.

The irreversible decline of membrane permeability is often due to strong adsorption of particles of contaminants onto the membrane surface and in its pores. In general, flux decline is caused by a decreasing driving force and/or an increased resistance.

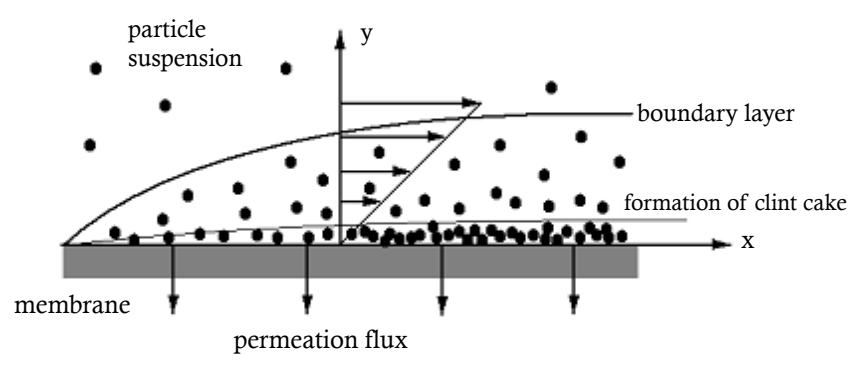

Figure 3: Formation of dirt cake on membrane due to concentration polarization. 
Citation: Loh WL, Wan TT, Premanadhan VK, Naing KK, Tam ND, et al. (2014) Experimental Study of the Separation of Oil in Water Emulsions by Tangential Flow Microfiltration Process. Part 1: Analysis of Oil Rejection Efficiency and Flux Decline. J Membra Sci Technol 5: 130. doi:10.4172/2155-9589.1000130

Page 4 of 6

Permeability $\left(\mathrm{cm}^{2}\right)$ versus Experiments Order

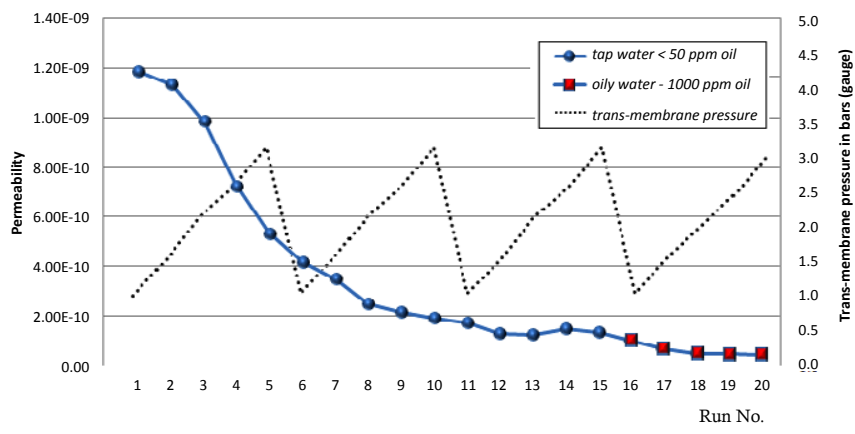

Figure 4: Permeability decline of ceramic membrane of $0.5 \mu \mathrm{m}$ pore size due to fouling.

Flow Resistance (bars.s/m) Versus Experiments Order

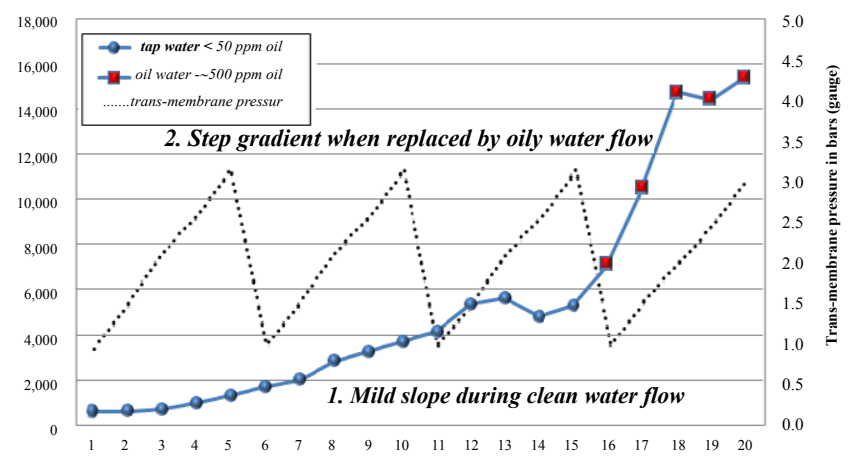

Run No.

Figure 5: Decrease of flow resistance on the ceramic membrane $0.5 \mu \mathrm{m}$ was observed when trans-membrane pressure were gradually increased from 1.0 to $3.5 \operatorname{bar}(\mathrm{g})$

As a series run mode was implemented, trans-membrane pressure was gradually increased from 1 to $3.5 \mathrm{bar}(\mathrm{g})$. The rapid decline in permeability indicates the higher the pressure, the greater decline in permeability were observed. After a nearly $80 \%$ decline in flux during first 10 runs of experiments, it finally reach a steady balance between the fouling and hydrodynamic force which constantly sweep away fouling materials from the membrane surface. Hence, a nearly steady permeability or constant permeability stage of approximately $1 \sim 2 \times 10$ $10 \mathrm{~cm}^{2}$ was achieved in the first experiment during the clean water feed phase (run no. 11 to 15), as shown in Figure 4.

With the gradual reduction in fouling, the flow permeability and filtration flux decreased slowly. When a steady permeability pattern is attained, the apparent effect of fouling is negligible. Thus, the flux is linearly dependent on the trans-membrane pressure alone. That's the reason oil-water filtration experiments were conducted from run no. 16 and onward, only then the effects of pressure on filtration quality and flux performance can be reasonably assessed, and a linear relation of pressure on the filtration rate can be established.

The flow permeability and resistance at run no. 15 were $1.33 \times 10-10$ $\mathrm{cm}^{2}$ and 5292 bar.s/m, respectively, as illustrated in Figures 4 and 5 . Once the experiments has transited from the clean water feed phase into the 1000 ppm oily water feed phase, the flow permeability immediately deteriorate thereafter. The resistance curve during the oil-water feed was showing a steeper climb (run no. 16 to 18 ) in Figure 5, that could mean an increased rate of fouling due to the existence of a secondary cake layer which may be caused by the organic (oil particles) fouling. The deposition of oil on the surface of membrane has actually trapped more suspended particles into the oil layer, therefore a "mixed" gel layer is formed, increases flow resistance and causes further decline in permeability. The next stable resistance at range around $15000 \mathrm{bar} . \mathrm{s} / \mathrm{m}$ was attained afterward, which was almost three times of the previous steady range (Figure 5).

Meanwhile, in another experiment using 500 ppm oily water feed, the flow resistance continues to soar until it reached a peak resistance of around 12000 bar.s/m, as depicted in Figure 6 .

This result is $21 \%$ lower than the peak resistance value as attained in the first experiment with $1000 \mathrm{ppm}$ of oil in water feed. Afterward, the resistance began to decline when the trans-membrane pressure reached beyond $1.5 \mathrm{bar}(\mathrm{g})$ range. The reason is to be explained later.

The measurements on permeate samples have been repeated five times for each sample and the analysis of statistical mean and standard deviation were carried out. The standard deviation $\sigma$ shows how much variation or "dispersion" exists from the average (mean, or expected value). In Table 2, a standard deviation of 1.78 means that most measurements (about 68\%, assuming a normal distribution) of permeate water samples have a reading within $1.78 \mathrm{ppm}$ of the mean. A relatively low value in the standard deviation indicated that high reproducibility was achieved (Table 2 ).

Table 3 shows a comparison of the filtrate quality in term of ppm of residual oil content in water after the microfiltration experiment with the clean water feed and the oil in water feed (Table 3).

According to equation (2-5), the results were later converted into oil rejection efficiency as depicted in Table 4.

Flow Resistance (bars.s/m) Versus Experiments Order

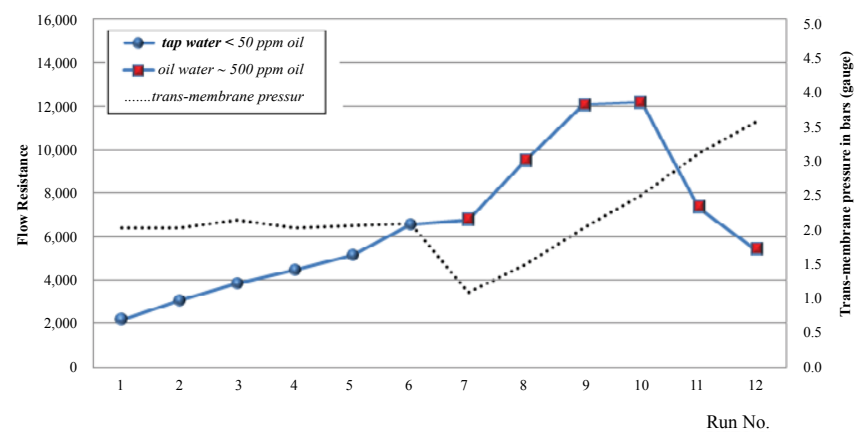

Figure 6: Increase of flow resistance on the $0.5 \mu \mathrm{m}$ pore size ceramic membrane due to fouling.

\begin{tabular}{|c|c|c|}
\hline Sample Description & Mean & $\begin{array}{c}\text { Standard } \\
\text { Deviation }\end{array}$ \\
\hline S09/P1.0/O1000 Permeate sample @ 1.0 bar(g) TMP & 35.9 & 1.00 \\
\hline S11/P1.5/O1000 Permeate sample @ 1.5 bar(g) TMP & 32.8 & 1.93 \\
\hline S13/P2.0/O1000 Permeate sample @ 2.0 bar(g) TMP & 35.7 & 0.82 \\
\hline S15/P2.5/O1000 Permeate sample @ 2.5 bar(g) TMP & 27.8 & 1.36 \\
\hline S17/P3.0/O1000 Permeate sample @ 3.0 bar(g) TMP & 95.3 & 3.38 \\
\hline S19/P3.5/O1000 Permeate sample @ 3.5 bar(g) TMP & 146.1 & 2.18 \\
\hline Average & 62.2 & 1.78 \\
\hline
\end{tabular}

Table 2: Mean and standard deviation for the measurement of oil concentration (in ppm) for permeate water samples collected from the microfiltration experiment using a ceramic membrane of $0.5 \mu \mathrm{m}$ pore size and oil-water feed of $1000 \mathrm{ppm}$. 
Citation: Loh WL, Wan TT, Premanadhan VK, Naing KK, Tam ND, et al. (2014) Experimental Study of the Separation of Oil in Water Emulsions by Tangential Flow Microfiltration Process. Part 1: Analysis of Oil Rejection Efficiency and Flux Decline. J Membra Sci Technol 5: 130. doi:10.4172/2155-9589.1000130

Page 5 of 6

\begin{tabular}{|c|c|c|c|c|c|c|}
\hline Feed concentration/TMP & $1 \operatorname{bar}(g)$ & $1.5 \operatorname{bar}(\mathrm{g})$ & $2.0 \operatorname{bar}(\mathrm{g})$ & $2.5 \operatorname{bar}(g)$ & $3.0 \operatorname{bar}(\mathrm{g})$ & $3.5 \operatorname{bar}(\mathrm{g})$ \\
\hline 1000 ppm oily water feed & 35.9 & 32.8 & 35.7 & 27.8 & 95.3 & 146.1 \\
\hline 500 ppm oily water feed & $<10$ & $<10$ & $<10$ & $<10$ & 35.1 & 72.0 \\
\hline
\end{tabular}

Table 3: Permeate quality (in ppm) from the microfiltration experiment using a ceramic membrane of $0.5 \mu \mathrm{m}$ pore size and oil-water feed of various concentration at different test pressure.

\begin{tabular}{|c|c|c|c|c|c|}
\hline Oil Rejection Efficiency (\%) /TMP in bar(g) & $\mathbf{1 . 0}$ & $\mathbf{1 . 5}$ & $\mathbf{2 . 0}$ & $\mathbf{2 . 5}$ & $\mathbf{3 . 0}$ \\
\hline During 500 ppm oily water feed & 98.78 & $>99$ & $>99$ & $>99$ & 92.98 \\
\hline During 1000 ppm oily water feed & 96.17 & 96.5 & 96.19 & 97.03 & 89.83 \\
\hline
\end{tabular}

Table 4: Oil rejection efficiency (\%) for microfiltration experiment using a ceramic membrane of $0.5 \mu \mathrm{m}$ pore size and oil-water feed of various concentration at different test pressure.

The permeate quality from the first filtration experiment with 1000 $\mathrm{ppm}$ feed concentration has the best results of $27.8 \mathrm{ppm}$ residual oil. The permeate quality were consistent until $2.5 \mathrm{bar}(\mathrm{g})$ pressure. However, the residual content has spiked up to 95.3 ppm oil @ 3 bar(g) pressure, and 146.1 ppm oil @ 3.5 bar $(\mathrm{g})$ pressure. The oil rejection efficiency were found to be maintained around 96 97\% before the pressure reached $2.5 \mathrm{bar}(\mathrm{g})$. At higher pressure range, oil phase began to break across the membrane. Therefore, a sudden decline in permeate quality has been observed. A similar finding has been observed when the feed was reduced to $500 \mathrm{ppm}$ oily water mixture in the next experiment. A lower feed concentration is definitely advantageous for attaining higher oil rejection efficiency. For the experiment with 500 ppm oily water feed, the permeate quality reduced to lower than $10 \mathrm{ppm}$ residual oil. Again, oil phase starts to break through the ceramic membrane of $0.5 \mu \mathrm{m}$ pore size when trans-membrane pressure exceeded $2.5 \mathrm{bar}(\mathrm{g})$.

The results indicated that trans-membrane pressure have negligible effects on the oil rejection efficiency before a critical pressure of 2.5 $\operatorname{bar}(\mathrm{g})$. As the suspended oil content of the feed increases, blockage of the pores increases and at a faster rate, which results in the growth of a thicker deposited layer. Therefore lower oil concentrations in the permeate samples should be expected when the feed contained a low concentration of suspended oils.

The previous comparison of peak resistance in both experiments indicates a significant improvement in the flow resistance $(\mathrm{R}=\Delta \mathrm{P} / \mathrm{J})$ at lower feed concentration. Higher feed concentration led to greater problem in fouling, therefore, higher flow resistance. As the transmembrane pressure increases, the tangential flow velocity has increased as well. Though with increased pressure and flow velocity it may have improved to some extent the degree of fouling, however, it came with a price of having more oil breaking through the membrane when the pressure exceeds $2.5 \mathrm{bar}(\mathrm{g})$. One likely explanation is the theory of deformable drops in capillaries due to pressure.

Nazzal et al. has suggested that the rejection of an oil drop was determined by its ability to deform and flow through a pore as permeate. He applied the Young-Laplace equation to propose a model for calculating the critical pressure required to force the entry of an oil drop into a membrane as follows [12]:

$$
P_{c}=2 \gamma \frac{\cos \theta}{r_{p}}\left[1-\left(\begin{array}{c}
\frac{2+3 \cos \theta-\cos ^{3} \theta}{4\left(r_{d} / r_{p}\right)^{3} \cos ^{3} \theta} \\
-\left(2-3 \sin \theta+\sin ^{3} \theta\right)
\end{array}\right)^{1 / 3}\right]
$$

where $\gamma$ is the interfacial tension, $r_{d}$ is the radius of the original drop, $r_{p}$ is the radius of the pore and $\theta$ is the contact angle. In his opinion, this critical pressure can be used for predicting the maximum operating pressure at which oil drops are not longer rejected by the membrane and their penetration leads to membrane fouling. This model is useful to interpret experimental effect of trans-membrane pressure on the microfiltration of oil in water emulsions. Initially, oil drop that does not wet the membrane cannot pass through a pore of smaller diameter. If the applied pressure is great enough beyond the threshold as predicted in equation (4-1), it will deform the drop and overcome surface tension effects within the membrane surface. The higher the applied pressure, it will tend to increase permeate flux and the flow of oil drops to another side of the membrane. The oil drops may either pass through the membrane, or possibly shear deeper into the interconnected pores and stuck inside the dead of the channel. As a results, blockage occur due to passage of oil drops through the membrane, where a constant decline in oil rejection efficiency is clearly revealed as pressure exceeds 2.5 bars $(\mathrm{g})$ and beyond. The threshold could be perceived as the critical pressure where oils will break through the pore in the ceramic membrane.

This observation was further supported by Davis in a simulation study of the micro scale flow of emulsions containing deformable drops through pores neck. He confirmed that whether a drop is able to deform enough the squeeze through the pore neck or not was depending on the capillary number. When the pressure across the membrane was sufficient, it exceeds the critical capillary number (a ratio of viscous deforming force and interfacial restoring forces) and would enable the drop to exceed the average velocity of the continuous phase and squeeze through the pore, otherwise, it would be trapped in a small pore neck [13].

Another explanation to the deterioration in the filtrate quality is due to the corresponding effects of turbulence as tangential flow velocity increases. During the experiment, the tangential flow velocity has increased with the trans-membrane pressure. He has reported that turbulence in flow have remarkably reduced the particle size in the filtration cake [14]. The reduction in particles size is definitely going to affect the quality of filtrate in a negative way. Finer particles and oil drops would find its way to enter and squeeze through the pore and pollute the water in the permeate side. Consequently, more oil would break through the membrane with a constant decline in oil rejection efficiency is clearly revealed.

\section{Conclusions}

The performance of microfiltration for the separation of oil-inwater emulsions has been investigated. At $1000 \mathrm{ppm}(0.1 \%)$ feed concentration, a microfiltration ceramic membrane of $0.5 \mu \mathrm{m}$ pore size was proven possible of producing a high purity filtrate lower than the threshold required for offshore produced water effluent, typically $29 \mathrm{mg} / \mathrm{l}$ (i.e. $33 \mathrm{ppm}$ ) residual oil in the Gulf of Mexico [15]. At 500 ppm $(0.05 \%)$ oil concentration, higher purity filtrate containing lower than $10 \mathrm{ppm}$ residual oil in permeate was proven possible at a transmembrane pressure not exceeding $2.5 \mathrm{bar}(\mathrm{g})$. The results attained were useful for evaluating the potential of tangential flow microfiltration process in the produced water treatment, with respect to the suitability to fulfill the regulatory requirement for disposal. 
Citation: Loh WL, Wan TT, Premanadhan VK, Naing KK, Tam ND, et al. (2014) Experimental Study of the Separation of Oil in Water Emulsions by Tangential Flow Microfiltration Process. Part 1: Analysis of Oil Rejection Efficiency and Flux Decline. J Membra Sci Technol 5: 130. doi:10.4172/2155-9589.1000130

Page 6 of 6

The study of the pressure effects on filtration capacity and permeability analysis is a very useful tool for analysis of changes in flux due to complicated influence of membrane pore size, pressure and fouling. The fouling study gives a practical estimation of the filtration capacity especially during the selection appropriate membrane for the need of various industrial treatment processes. The method is also used to optimize the separation efficiency while having the best cleaning ability by hydrodynamic tangential flow.

However, the major challenge of operation is the control of fouling. This research does indeed look at the separation and fouling control as a system instead of an individual subsystem, with the results contributing primarily to the knowledge base of separation with a secondary contribution to the fouling control problem. For the objective of controlling the fouling, a series of studies have been conducted on insitu cleaning to allow remediation of polluted surface during filtration. The result of the study will be presented in the next paper.

\section{References}

1. Fakhru'l-Razi A, Pendashteha A, Abdullaha LC, Awang Biaka DR, Madaenic SS, et al. (2009) Review of technologies for oil and gas produced water treatment. Journal of Hazardous Materials 170: 530 -551.

2. Mueller J, Cen Y, Davis RH (1997) Crossflow microfiltration of oily water Journal of Membrane Science 129: 221-235.

3. Holdich RG, Cumming IW, Smith ID (1998) Crossflow microfiltration of oil in water dispersions using surface filtration with imposed fluid rotation. Journal of Membrane Science 143: 263-274.

4. Cheryana M, Rajagopalan N (1998) Membrane processing of oily streams Wastewater treatment and waste reduction, Journal of Membrane Science 151: 13-28.
5. Koltuniewicz, AB, Field RW (1996) Process factors during removal of oil-inwater emulsions with cross-flow microfiltration. Desalination 105: 79-89.

6. Chen ASC, Flynn JT, Cook RG, Casaday AL (1991) Removal of oil, grease, and suspended solids from produced water with ceramic cross flow microfiltration. SPE Prod Eng 6: 131-136.

7. Arnot, TC, Field, RW, Koltuniewicz AB (2000) Cross-flow and dead-end micro filtration of oily water emulsions Part II. Mechanisms and modeling of flux decline. Journal of Membrane Science 169: 1-15.

8. Dejak M (2013) Keeping water soft. Oilfield Technology 6: 35-44.

9. Al-Malack MH, Anderson GK (1997) Use of cross flow microfiltration in wastewater treatment. War Res 31: 3064-3072.

10. Rajinder Pal (1994) Techniques for measuring the composition (oil and wate content) of emulsions - a state of the art review. Colloid and Surface A. Physicochemical and Engineering Aspects 84: 141-193.

11. Environment Protection Agency (1999) Method 1664, Rev. A: N-hexane extractable material (HEM; oil and grease) and silica gel treated $n$-hexane extractable material (SGTHEM; non-polar material) by extraction and gravimetry. EPA-821-R-98-002.

12. Nazzal FF, Wiesner MR (1996) Microfiltration of water-in-oil emulsions. Water Environment Research 68: 1187-1191.

13. Davis RH (2009) Motion of deformable drops through granular media and othe confined geometries. Journal of Colloid and Interface Science 334: 113-123.

14. Gaohong He (2012) A comparison of cake properties in traditional and turbulence promoter assisted microfiltration of particulate suspensions. Water Research 46: 2535-2544

15. Stewart M, Arnold K (2009) Emulsions and Oil Treating Equipment : Selection, Sizing and Troubleshooting. Gulf Professional Publishing, USA, UK. 107-211. 